\title{
On the Motivation of Language and Expression in the Chinese language
}

\author{
Shou Yan MIAO ${ }^{1,2}$ \\ 1) School of Liberal Arts, NanJing University, NanJing, JiangSu, China. \\ 2) School of Liberal Arts, Linyi University, Linyi, Shandong, China.
}

\begin{abstract}
The language symbols can be proved and has the very strong motivation that is both direct or indirect . Iconicity is the most important form of Motivation, it is the simulation relation of the language structure and cognitive structure, or is the relationship between language forms and features of the objective world. it can also be a link between the source words and new words. The image of Iconicity is the psychological image, but not is the object image. The Chinese language has the very strong motivation, the motivation can be detected four aspects of language. such as phonetic, characters, grammar. Syntactic iconicity is the most obvious.
\end{abstract}

Keywords—language symbols, Motivation, Iconicity, Chinese language

\section{语言符号的理据性及在汉语中表现}

\author{
苗守艳 ${ }^{1,} 2$ \\ 1) 南京大学文学院, 南京, 江苏, 中国 \\ 2) 临沂大学文学院, 临沂, 山东, 中国
}

摘 要 语言符号的产生是可以论证的, 具有很强的理据性, 既有直接理据也有间接理据。理据性可以是语言形式与客观事物特 征之间联系, 也可以是源生词和后起词之间联系。象似性是理据性最重要表现形式, 是指语言结构与人类认知结构的映祄、模拟等内 在联系。象似性的 “象” 是 “心象”, 不是简单的 “物象”, 更不是具体相似镜像反映。汉语也具有极强的理据性, 主要表现在语音、 文字、语法、语篇四个层面，其中语法象似性表现最明显。

关键词 语言符号, 理据性, 象似性, 汉语

\section{1. 引言}

近些年随着认知语言学的兴起, 语言符号的象似性成 为关注的热点。但大部分研究者都是把象似性看做理据性 且是与任意性相对的概念来阐述研究, 而忽略了象似性与 理据性差异。目前国内语言学界对语言符号的理据性研究 存在两方面问题: 一是对语言符号理据性界定不清楚; 二是 对汉语符号理据性缺乏明确的论证。本文从语言符号理据 性的内涵界定入手, 分析理据性重要表现形式, 并明确了 汉语符号的理据性。

\section{2. 语言符号的理据性}

\section{1 理性性不同于象似性}

象似性是与理据性长期相混一个概念, 讨论语言符号 理据性不可避免要涉及象似性这个术语。最初是哲学家们 讨论 “词是否可以看作是真实物体与生命体的图象（模 仿)”，他们关心的象似性是语言起源的本质问题，即语言 是第一性还是物质第一性。19 世纪洪堡特和维特根斯坦提 出语言与现实同构的观点, 认为语言是对客观世界的模仿。 维特根斯坦把语言本性归结为 “图象理论”, 认为图象摹画 世界。后来符号学家把象似性作为重要参数引进符号学中, 19 世纪皮尔斯把符号分为 “symbol”、“index” 和 “i con” 其中他提出的第三类符号 “i con” 象似符, 指那些表达与 
对象有一定相似性的符号。他并没有把 “图象” 的使用限 制在对某个对象或多或少是现实的, 非语词的模仿上, 或 者在语言层面上, 也不限制在语音-象征的表达 [1], 而是 突破性地开始了语言象似性研究。后来认知语言学家则把 语言象似性研究推向了高潮, 他们认为语言是对现实世界 经过人类认知加工而形成的, 在现实和语言之间存在 “认 知” 这个中介, 认知先于语言、决定语言、是语言的基础, 人类对现实世界感知体验和认知加工基础上形成了概念结 构。语言形式通过概念结构与客观现实之间存在对应性象 似现象, 即是 “心象” 而不是 “物象” 的模拟, 更不是具 体形状的镜像 “相似”。由此可以看出象似性内涵在不同领 域有差异, 哲学家的象似性侧重的是 “摹画”, 符号学家象 似性强调的是 “图象”, 语言学家强调象似性是 “映射”。

语言象似性成为反对语言任意说学者使用的一个概 念, 国内一些学者如沈家煊先生、王寅先生、严辰松他们 就把象似性定义为 “非任意性”, 再把它直接等同于 “理据 性”。但象似性并不等同于理据性, 象似性是语言形式与意 义之间更为具体的理据关系, 即指语言结构与人类认知结 构的映祄、模拟等内在联系。当语言与表现形式不同质, 但语言总能与表现形式之间找到联系, 语言的理据性甚至 模糊得难以辨认, 就是间接象似。理据性是指表达语言单 位或语义范畴形式选择的理由和根据, 包括词据、概念理 据、义类理据、观念实现方式、意念取象、句义表达式等 [2]。语言形式上反映了表达的意义, 就表现为理据性, 如 果语言形式通过模拟意义来反映后者, 从而造成两者相似, 那就表现为象似。象似性一定是有理据的, 但有理据的不 一定是象似的。比如, 语言中概念转喻现象都是有理据的, 如水手指船员, 但语言形式和表达的 “人” 意义并不相像 ${ }^{[3]}$ 。我们可以看到象似性和理据性差异, 象似性不等于更 为广义的 “非任意性”, 它只是理据性表现形式之一。

\section{2 理据性内涵界定}

语言任意性说者坚持认为语言形式和客观事物没有任 何相似之处, 从而反对语言理据性。这是因他们对理据性 有误解而造成。首先误解 “理据性” 等同于 “象似性”。象 似性强调的是语言形式通过认知模式与客观现实的模拟, 这只是理据性一种表现形式而已, 还可以有隐喻、转喻等 形式。其次误解 “理据性” 的语音论。认为理据性一定是 指语言声音和真实世界事物之间的联系, 实际上理据性既 可以指声音和意义之间联系, 也可以指事物特征与意义之 间联系, 甚至可以是源生词与后起词之间的联系。可能声 音和意义之间联系更直接, 易于辨认, 而源生词和后起词 之间联系更间接模糊而已。
我们对语言理据性内涵概括如下：(1)理据性是与任意 性相对的概念, 指语言符号所指和能指之间具有联系, 不 是任意的。(2)理据性不仅指声音与意义之间联系, 还可以 是客观事物特征与语言形式之间联系, 甚至还可以是源生 词的声音、特征与后起词之间联系。(3)理据性中最重要表 现形式是象似性, 强调的是语言与客观事物之间通过 “心 象” 而不是 “物象” 的模拟, 是语言与表现形式之间关系 的映射, 而是不一种镜像的相似。

认知语言学家构建了语言源于表达现实世界的需要的 理论。虽有学者认为这用 “图象、反映” 来理解语言的产 生是极为浅俗的, 但我们认为却比较恰当。先民在劳动中 呼喊传递信息, 发现了可用不同声音符号表述不同事物和 事件, 其中大部分是模拟大自然声音, 有明显理据性。这 些声音符号被不断分解、重组从而表达更抽象更完整概念。 从这个角度说, 语言产生的参照物可以从言内、言外、言 际去寻找, 语言理据性就有了更广阔的涵义。

\section{3. 汉语符号理据性的表现}

汉语是汉民族语言, 同样具有明显的理据性特点。我 们主要从词语产生、词语组合角度谈汉语理据性, 主要表 现在语音、文字、语法、语篇等方面, 其中语法象似性最 为明显。

\section{1 语音理据性}

语音象似性（有象似性自有理据性）最典型实例莫过 于象声词 (主要是拟声词和叹词)。从语言系统角度看, 叹 词属于比语言系统原始的第一符号系统, 是类语言; 拟声 词是人类语言符号重要一部分, 拟声词系统内所有侧面及 各侧面上的成分都服从于语言系统的规则制约。汉语拟声 词的象似性是非常明显并被大家认可, 其他词类的象似性 往往引起大家争论。目前研究表明名词、动词、形容词等 词类及其中很多词汇均在拟声词和拟形词基础上杽乳出来 ${ }^{[4]}$ 。那么, 大部分词语也就具有了理据性。

古汉语经典《伐檀》有 “坎坎伐檀兮、置之河之干兮”, 很能说明古汉语词汇中词语的音义之间联系。古人注意到 了语音和语义的不可分割性, 才有了 “因声求义” 训诂方 法。先秦时期, 如《孟子 - 滕文公上》: “痒者养也, 校者 教也, 序者射也。”, 到了汉代因声求义得到推广, 如《诗・围米 风・东山》 “丞在栗薪”, 《郑笺》: “栗, 析也……, 古者声, ” 栗、裂同也。晋代以后则发展为 “右文说”。到了清代, 段 玉裁提出 “圣人之治字有义而后有音, 有音而后有形。学 者之考字, 因形以得其音, 因音以得其义, 治经莫重于得 义, 得义莫切于其音”。王念孙在《广雅疏证》自序中提出 
“窃以训诂之旨, 本于声音。故有声同字异, 声近义同, 虽或类聚群分, 实亦同条共贯。今则就古音以求古音, 引 伸触类, 不限形体”。戴震《转语二十章序》: “疑于义者以 声求之, 疑于声者以义正之。” 学者对因声求义有了清楚 的认识, 解决了很多词语释义问题。因声求义的训诂方法 恰是汉语语音理据性的表现。

汉语声调也表现出理据性。在小学语文教学中, 小学 生在声调学习中, 读不同声调会随声调的升降而有形体的 变化, 这应该是拟形理据造成的。我们可以认为, 人民出 于某种微妙心理联想过程, 总是用某些声音来表现相类似 事物特征, 这也是一种语言符号象似性表现。

\section{2 文字理据性}

语言和文字是虽是两个系统, 但却密切相关, 我们往 往都是把书面语言的文字等同于语言, 特别是文学语言中, 更多是指文字。文字是形音义三者结合, 是语言的记录者, 文字形体本身就具有象似性, 文字学中 “图画文字” 的概 念正是语言象似性说明。众多的文字学家都认为文字起源 与图画有关。不论是表意文字还是表音文字都能找到起源 与图画的事实材料。(现在可考的表音文字也都是直接或间 接来源于腓尼基文字, 而腓尼基文字也是来源于图画。）许 慎认为文是对物象描摹, 而字则是象形的符号和象声的符 号复合而成。文字起源于图画, 而图画是模仿现实世界, 这也说明了文字具有直接或间接的象似性。

古汉语中 “词” 与 “字” 的界限一直不十分明确, 古 汉语中大部分字都可以看做词, 古汉语字词有 “音生于义, 义著于形”, ${ }^{[5]}$ 特征, 故有了 “据形释义” 的训诂方法。许 慎六书中对 “象形、指示、会意” 界定说明了字形和意义 之间具有密切关系。汉语中百分之八十的形声字, 研究表 明不论是声兼义字还是声不兼义类的形声字, 声符和字义 之间都有密切关系, 所以说汉语的文字也表现出很强的理 据性。

\section{3 语法理据性}

语法象似性（理据性）表现如下:

(1) 语序象似性。语言符号之间组合是线性的, 这种线 性排列顺序适合客观世界中事件发生的先后顺序之间有关 联, 这就表现为语序象似性, 句法成分的排列顺序映照它 们所表达的交际状态或事件发生的先后顺序 ${ }^{[6]}$, 如: “我走 进教室, 打开书包, 拿出文具盒。” 古汉语语序同样遵循这 个顺序象似性, 如: “孟尝君使人给其食用, 无使乏, 于是 冯谖不复歌。(战国策 - 齐策四)”。再如: “吾不能早用子, 今急而求子, 是寡人之过也。” 这种主谓宾结构反映了思维
上的顺序，只有在特殊句式才不遵循此原则。汉语中语序 与时序存在较高象似性, 它们如同 “临摹式” 的绘画一样, 语序直接反映生活。另外汉文化中 “先上后下, 先尊后卑” 思维定势决定了汉语的词序, 如 “左顾右盼, 七上八下, 高不成、低不就, 喜新厌旧” 等, 这样的词序也是语法理 据性表现。

(2) 数量象似性。语言形式和意义之间数量存在一定的 象似性, 即语言表达时, 概念上容量大、内容重要, 难以 预测的信息, 表达方式会复杂, 相反则言简意赅。数量象 似性与重复, 赘言 ( 冗言); 排比, 双关; 省略; 词汇密度, 信息密度; 简单句, 复合句, 长句, 短句; 曲言等密切相 关。 ${ }^{[7]}$ 李清照名句 “寻寻受受, 冷冷清清, 凄凄惨惨戚戚” 重叠的语句和诗人凄苦的心情象似。不仅句法层面上有数 量象似性, 在词汇层面同样也具有数量象似性, 汉语中并 列结构较多也是数量象似性的表现。

（3）标记象似性。标记性从无到有象似于认知的自然顺 序, 带有区别性特征的有标记的语言成分比没有标记的成 分具有额外的、不寻常的意义。[7]词汇层面上, 基础范畴 的词是无标记的, 其他层次的则是有标记的, 比如 “牛” 是基础范畴词语, 没有标记, 但 “犀牛、黑牛” 等则属于 有标记; 句法层面上, 陈述句是无标记, 但疑问句、否定 句等则属于有标记, 主动句是无标记的, 把字句、被字句 则都属于有标记的句式; 口语是无标记, 书面语则是有标 记的。

\section{4 语篇理据性}

语篇形式和意义之间关系密切, 体现了语篇的理据性。 语篇的中理据性主要表现在语音、形状、时间序列、空间 序列等方面, 比如通过声调、声韵的运用制造紧张、舒缓 等效果。语篇可以通过书写形式特意安排临摹事物, 如近 几年流行的图形诗、具象诗、图案诗、棒状诗、修剪诗、 排版诗, 通过文字本身的字形或者透过排列组合文字符号, 在外形上引起特殊的效果。语篇理据性还表现在意识流小 说中, 通过词语的跳跃、缺乏连贯模拟人物的意识跳动奔 流, 因为语言逻辑的断续、错位和裂变, 正是人类思维活 动的外在化。语篇的象似性是语音象似、文字象似、语法 象似的综合运用。通过各种象似手段, 实现各自文体的文 体特征。

\section{4. 结语}

研究表明语言符号产生是可以论证的, 具有很强的理 据性 (象似性), 语言符号的理据性是与语言符号任意性相 对立概念, 属于语言产生的内因。语言产生策略是建立在 
拟音拟形的基础上, 依靠各种理据策略产生。理据性可以 是语言结构与人类认知结构的映祄、模拟等内在联系, 也 可以是语言形式与客观事物特征之间联系, 也可以是源生 词和后起词之间联系。既可以是音义之间关联, 也可以是 形义之间, 甚至是义义之间关联。象似性是理据性的重要 形式, 很多情况下, 象似性与理据性等同使用, 但象似性 不是理据性唯一形式。语言的理据性的是以认知心理上的 相似、接近、顺接、凸显等原形认知为基础, 构成了语言 的顺序象似、距离象似性、标记象似性等。汉语言符号在 语音、文字、语法、语篇四个层面上表现出极强的理据性。

\section{参考文献(References)}

[1] Ungerer. F. Schmid. H. J, An Introduction to cognitive Linguistics -Second Edition. Shanghai: Fudan University press, 2009: 342.
[2] MA Qinghua, Cultural Semantics. Nanchang: Jiangxi people's Publishing House, 2006: 35.

[3] LIFuyin, An introduction to cognitive linguistics. Peking: Peking University Press, 2008: 45.

[4] MA Qinghua A Study Toward the Primitiveness of the Relationship Between Interjection's Form and Meaning. Linguistic Sciences, 2011 (05).

[5] $\mathrm{Su}$ Baorong,SongYongPei. Introduction to ancient Chinese meaning. ShiJiazhuang: Hebei education publishing house, 1987: 46.

[6] Shen Jiaxuan, A survey of studies of iconicity in syntax, Foreign Language Teaching and Research, 1993 (01).

[7] Wang Yin. M arkedness Iconicity. Foreign Language Research, 1998 (03). 